Linkou Chang Gung Memorial Hospital were collected. Study subjects were those: 1 ) with known endometrial lesions, which including any type of endometrial hyperplasia and any stage of endometrioid carcinoma of the endometrium, 2) with symptoms/signs of endometrial hyperplasia or endometrial cancer, including abnormal uterine bleeding with thick endometrial stripe or medically uncontrollable uterine bleeding, as study group. Patients with benign uterine/ovarian tumors were invited as the control group. The sample was collected using a designed cotton swab by study physician and was stored in ?? $0^{\circ} \mathrm{C}$ until ELISA analysis.

Results: Statistically significant elevation of IL-6 titer and VEGF titer but not PRTN-3 among endometrial cancer patients, compared with those with normal uterus or with benign tumors, were noted. No significant differences were observed for PRTN-3 level within the groups. Algorithm for endometrial hyperplasia and endometrial cancer screening is established accordingly.

Conclusion: This study represents the first evaluation of IL-6, PRTN-3 and VEGF level extracting from vaginal fluid as potential markers in screening for endometrial cancer. As a noninvasive measure, further validation study is needed.

Oral (OMi5)

Gynecologic Pathology, Genetics and Epidemiology

https://doi.org/10.3802/jgo.2021.32.S1.0Mi5

\section{Association between hospital treatment volume and survival of surgical cases with gynecologic malignancy in Japan}

\section{Hiroko Machida, ${ }^{1}$ Koji Matsuo, ${ }^{2}$ Daisuke Aoki, ${ }^{3}$ Takayuki Enomoto, ${ }^{4}$ Aikou Okamoto, ${ }^{5}$ Hidetaka Katabuchi, ${ }^{6}$ Satoru Nagase, ${ }^{7}$ Masaki Mandai, ${ }^{8}$ Nobuo Yaegashi, ${ }^{9}$ Mikio Mikami ${ }^{1, *}$}

'Tokai University School of Medicine, Isehara, Japan (mmikami@is.icc.utokai.ac.jp)

${ }^{2}$ University of Southern California, Los Angeles, CA, USA

${ }^{3}$ Keio University School of Medicine, Tokyo, Japan

${ }^{4}$ Niigata University School of Medicine, Niigata, Japan

${ }^{5}$ Jikei University School of Medicine, Tokyo, Japan

${ }^{6}$ Kumamoto University, Kumamoto, Japan

${ }^{7}$ Yamagata University Faculty of Medicine, Yamagata, Japan

${ }^{8}$ Kyoto University Graduate School of Medicine, Kyoto, Japan

${ }^{9}$ Tohoku University, Sendai, Japan

Objective: Associations between hospital treatment volume and survival outcomes for surgical cases with 3 types of gynecologic malignancies, and the trends and contributing factors for highvolume centers were examined.

Methods: The Japan Society of Obstetrics and Gynecology tumor registry data based retrospective study examined 167,642 women with 76,255, 41,187, and 45,200 of endometrial, cervical, and ovarian tumor, respectively, who underwent primary surgery in Japan between 2004 and 2015. Associations between the annual treatment volume and overall survival (OS) for each tumor type were examined. Institutions were categorized into 3 groups (low-, moderate-, and high-volume centers) based on prior study.

Results: Among the high-volume centers, the median surgical treatment volumes were 67 (interquartile range [IQR], 59-82), 68 (IQR, 59-91), and 35 (IQR, 31-41) cases/year for endometrial, cervical, and ovarian tumors, respectively. On multivariate analysis, younger age, rare tumor histology, and lymphadenectomy were contributing factors for women at high-volume centers (all, $\mathrm{p}<0.001$ ). During the study period, the proportion of high-volume center treatments was decreased, whereas that of low-volume center treatments was increased for the 3 tumor types (all, $\mathrm{p}<0.001$ ). Surgical treatment at high-volume centers improved OS than that at other centers (adjusted-hazard ratio [95\% confidence interval]: 0.82 [0.760.88 ], 0.83 [0.76-0.91], and 0.88 [0.84-0.93] for endometrial, cervical, and ovarian tumors, all, $\mathrm{p}<0.001)$.

Conclusion: For women with gynecologic malignancy in Japan, surgical treatments at high-volume centers conferred survival benefits. The practice pattern shifted with scattering of patients and treatments at high-volume centers were decreasing.

Oral (OMi6)

Gynecologic Pathology, Genetics and Epidemiology

https://doi.org/10.3802/jgo.2021.32.S1.0Mi6

\section{Mutations of DNA damage repair pathway- related genes in ovarian caner detected by next-generation sequencing}

\author{
Rong Feng, Shoutai Ding, Huiling Chen, Chaoran Xia, \\ Zhixiang Yan, Peng Luo, Jiajia Xu" \\ Topgen Biological Medicine Technology Co., Ltd., Shanghai, China \\ (xujiajia@topgen.com.cn)
}

Objective: To study the mutations occurred in related genes in DNA damage repair pathway (DDR) in ovarian cancer (OC) patients detected by next-generation sequencing (NGS). Methods: After NGS of tissue samples from 205 ovarian cancer cases, the mutation types and mutation frequencies of DDR pathway-related genes were classified and analyzed. Two-hundred five female Chinese patients with OC (including primary peritoneal cancer and fallopian tube cancer) were involved and detected by OncoDrug-Seq ${ }^{\mathrm{TM}} 603$-gene panel assay through NGS using Illumina NovaSeq 6000.

Results: The mutated genes in the 205 OC patients were 